\title{
Epidemiology and outcome of methicillin-resistant Staphylococcus aureus health-care associated pneumonia* $^{*}$
}

\author{
Hadeel Zainah ${ }^{1 \#}$, Syed Hassan ${ }^{2}$, Rasha Nakhleh ${ }^{3}$, Samia Arshad $^{1}$, Marcus Zervos $^{1}$ \\ ${ }^{1}$ Division of Infectious Diseases, Henry Ford Health System, Detroit, MI, USA; ${ }^{\#}$ Corresponding Author: hzainah1@hfhs.org \\ ${ }^{2}$ Department of Internal Medicine, Henry Ford Health System, Detroit, MI, USA \\ ${ }^{3}$ Department of Internal Medicine, Hurley Medical Center, Flint, MI, USA
}

Received 23 January 2013; revised 28 February 2013; accepted 7 March 2013

\begin{abstract}
Objectives: Methicillin-resistant Staphylococcus aureus (MRSA) is an important cause of healthcare-associated pneumonia (HCAP). HCAP confers high morbidity and mortality. In this study, we evaluated risk factors and clinical outomes of MRSA-HCAP. Methods: Between 08/2008 and 09/2010 we conducted a retrospective, singlecenter study. Seven hundred and eighty three patients with MRSA isolated from respiratory cultures were screened. The primary outcome was 28-day mortality. Results were computed using univariate and multiple logistic regression analysis. Results: The analysis included 63 patients (54.78\%) with MRSA HCAP. The mean age ( $\pm S D$ ) was $65.95 \pm 15.4$ years. Thirty seven $(58.7 \%)$ were males. Fifty six (88.9\%) had at least one associated comorbid medical condition. Forty two (66.7\%) were treated with vancomycin, 4 (6.3\%) with linezolid and the remaining $17(27 \%)$ were switched between the two antimicrobials. Mean APACHE II ( $\pm S D)$ score was $18.8( \pm 7.3)$. Mortality rate was $28.6 \%$. APACHE-II score, chronic obstructive pulmonary disease (COPD) and superinfection were found to be independent risk factors for 28-day mortality. Conclusions: MRSA is a significant etiology of HCAP which is more prevalent in the elderly. The associated mortality rate could be high and driven by several factors including APACHE II score and preexisting COPD.
\end{abstract}

Keywords: MRSA; Healthcare-Acquired;

Pneumonia; Epidemiology; Outcome

\section{INTRODUCTION}

Methicillin-resistant Staphylococcus aureus (MRSA) is

${ }^{*}$ No funding was received for this study. one of the leading causes of healthcare-associated pneumonia (HCAP) in the United States, accounting for $20 \%$ - $40 \%$ of cases [1]. It is known to cause significant morbidity and mortality complicating the hospital course and prolonging stay with an overall increase in healthcare cost.

The term HCAP was first incorporated in the 2005 nosocomial pneumonia guidelines by the American Thoracic Society and the Infectious Diseases Society of America (ATS/IDSA) to increase awareness of risk factors, organisms and treatment guidelines for these infections [2]. Most of the earlier studies however have focused on hospital-acquired and ventilator-associated pneumonia (VAP). Early studies of HCAP include a heterogeneous group of patients, partly because of differences used in definitions $[3,4]$. In this study therefore attempted to further define the risk factors associated with MRSA HCAP and clinical outcomes of the infection. Although there is much information on the epidemiology and measures for prevention of hospital acquired pneumonia (HAP); a better understanding of risk factors and outcome of HCAP is essential for the design of better prevention, diagnosis and management measures.

\section{MATERIALS AND METHODS}

This is a retrospective, single center study carried out at Henry Ford Hospital, a 900-bed tertiary-care teaching hospital located in urban Detroit, Michigan. All patients (No. 783) with respiratory cultures positive for MRSA identified from the hospital's microbiology database from 08/2008-09/2010 were eligible for inclusion. A detailed review of the medical records was carried out; patient's $\geq 18$ years old who met the ATS/IDSA defined criteria of HCAP were included in the study.

Pneumonia was defined by the presence of a new or evolving pulmonary infiltrate found on chest radiography along with two or more of the following criteria: production of purulent sputum or change in sputum character; 
fever $\geq 38^{\circ} \mathrm{C}$ or hypothermia $\leq 35.5^{\circ} \mathrm{C}$; total leukocyte count $>10,000 / \mu \mathrm{L}$ with $>15 \%$ increase in the absolute neutrophil count.

HCAP was defined in patients who were hospitalized for more than 2 days within 90 days prior to the diagnosis of pneumonia, nursing home or long term-care facility residents, on peritoneal or hemodialysis, undergoing intravenous antibiotic therapy or chemotherapy or receiving wound care within 30 days of admission.

The antibiotics used for the treatment were intravenous (IV) vancomycin, IV or oral linezolid or a switch between the two agents at the discretion of the physician caring for the patient. Treatment duration included the number of days needed to treat HCAP starting at initiation of therapy. MRSA was isolated from the sputum, tracheal aspirates or bronchoalveolar lavage (BAL).

Acute Physiology and Chronic Health Evaluation (APACHE II) score was used to grade the severity of illness. Any infection diagnosed within 48 hours of HCAP diagnosis was defined as co-infection, while infections seen after 48 hours were termed superinfections. The primary outcome was 28 -day mortality.

Univariate analysis was used to compare the baseline characteristics of the patients; comparison was done between those who survived up to 28 days versus those who did not. Categorical variables were expressed as percentages and absolute values; they were compared using Chi Square test, Fischer Exact test or Cochran-Armitage Trend test. Continuous variables were expressed as means \pm standard deviation and the results were calculated using Two sample T-Test. Variables in the univariate analysis with a $\mathrm{p}$-value $<0.15$ were included in multivariate analysis. Multiple logistic regression models were used to independently analyze each variable in predicting 28-day outcome (all cause mortality). A p-value $<0.05$ was considered statisticalyl significant. Statistical analysis was carried out using Stata 10.0 software (StataCorp, College Station, TX). This study was approved by the Institution Review Board at Henry Ford Health System.

\section{RESULTS}

Between August 2008-December 2010, the records of 783 patients with MRSA isolated from respiratory cultures were reviewed. One hundred and fifteen patients were found to have pneumonia, among which 64 (55.7\%) met the criteria for healthcare-associated infection but one patient was excluded from the analysis due to lack of information in the chart, 18 (15.7\%) had HAP, 23 (20.0\%) had VAP and 10 (8.7\%) had community-acquired pneumonia. Baseline characteristics and risk factors of the patients are illustrated in Table 1. The mean age $( \pm \mathrm{SD})$ was $65.95 \pm 15.4$ years. Thirty seven $(58.7 \%)$ were males. Fifty six $(88.9 \%)$ of them had at least one associated comorbid medical condition. Forty two $(66.7 \%)$ were treated with vancomycin, $4(6.3 \%)$ with linezolid and the remaining $17(27 \%)$ were switched between the two antibmicrobials. Mean APACHE II $( \pm \mathrm{SD})$ score was 18.8 $( \pm 7.3)$.

MRSA isolates were obtained from sputum in 44 $(69.84 \%)$ of the cases, followed by tracheal aspirate in 11 $(17.5 \%)$ and the remaining $8(12.7 \%)$ were obtained from BAL. Around $88.9 \%$ of the patients had at least one comorbid condition, the most common were diabetes and vascular disease (peripheral and central). Of the 63 patients with MRSA HCAP included in the analysis, 9 (14.3\%) had coexisting MRSA bacteremia. The average length of hospitalization $( \pm \mathrm{SD})$ was $19.1 \pm 17.6$ days; while the intensive care unit length of stay $( \pm \mathrm{SD})$ was $13.1 \pm 18.0$ days. Forty one patients $(65.1 \%)$ required mechanical ventilation. The mean APACHE-II $( \pm$ SD) score was $18.8 \pm 7.3$.

Twenty-eight-day mortality rate was $28.6 \%$. The predominant minimum inhibitory concentration (MIC) to vancomycin was $1 \mu \mathrm{g} / \mathrm{ml}$ (Table 1). Empiric treatment for gram negative organisms was started in all the patients but was deescalated in 34 patients (54.0\%). When we compared baseline characteristics, APACHE II score was the only difference between survivors and nonsurvivors (Table 2). However, APACHE II score, chronic obstructive pulmonary disease (COPD) and the presence of superinfection predicted 28-day mortality (Odds ratio was 1.153 (1.017 - 1.307), 7.869 (1.387 - 44.646), and 14.057 (1.532 - 128.982) respectively) (Table 3). There were no differences in outcome by the treatment used, MIC value to vancomycin or de-escalation of therapy.

\section{DISCUSSION}

Our study revealed that 28-day mortality rate associated with MRSA-HCAP was $28.6 \%$. Furthermore, APACHE II score, chronic obstructive pulmonary disease (COPD) and the presence of superinfection predicted 28day mortality. The diagnosis of pneumonia was uncommon in patients from whom a positive culture for MRSA was identified from respiratory specimens, as only 115 (14.68\%) met criteria for diagnosis.

2005 IDSA/ATS guidelines introduced the definition of HCAP in reference to the patients in whom pneumonia develops outside of the hospital, however have been in contact with a health-care setting and suspected to harbor multi-drug resistant organisms. Following which several studies have reported higher incidence of mortality in this population [5-7]. Currently there is a debate over the definition of HCAP; efforts are being made to refine the definition $[8,9]$. The Center of Diseases Control and Prevention (CDC) exclusively classifies infections as healthcare-associated or community-associated infections based on the presence of healthcare risk fac- 
Table 1. Patients' baseline characteristic.

\begin{tabular}{|c|c|}
\hline Variable & $\begin{array}{c}\text { Results } \\
\text { (Total number of patients: 63) }\end{array}$ \\
\hline Age (years), mean $\pm \mathrm{SD}$ & $65.95 \pm 15.4$ \\
\hline Sex, male, N (\%) & $37(58.7 \%)$ \\
\hline \multicolumn{2}{|l|}{ Underlying medical condition } \\
\hline Bronchiectasis, $\mathrm{N}(\%)$ & $4(6 \%)$ \\
\hline Active Malignancy, N (\%) & $10(16 \%)$ \\
\hline AIDS, N (\%) & $2(3 \%)$ \\
\hline End Stage Liver Disease, N (\%) & $7(11 \%)$ \\
\hline End Stage Renal Disease, N (\%) & $8(13 \%)$ \\
\hline COPD, N (\%) & $17(27 \%)$ \\
\hline Congestive Heart Failure, N (\%) & $21(33 \%)$ \\
\hline Diabetes Mellitus, N (\%) & $25(40 \%)$ \\
\hline Vascular disease, $\mathrm{N}(\%)$ & $26(41 \%)$ \\
\hline Use of steroids, $\mathrm{N}(\%)$ & $3(4 \%)$ \\
\hline Antibioticexposure 30 days prior to pneumonia diagnosis, $\mathrm{N}(\%)$ & $20(32 \%)$ \\
\hline Hospitalization for greater than 5 days before treatment initiation, $\mathrm{N}(\%)$ & $10(16 \%)$ \\
\hline Multilobar pneumonia, N (\%) & $24(38 \%)$ \\
\hline Length of hospital stay (days), mean \pm SD & $19.1 \pm 17.6$ \\
\hline Length of ICU stay (days), mean \pm SD & $13.1 \pm 18.0$ \\
\hline Mechanical ventilation (days), mean $\pm \mathrm{SD}$ & $7 \pm 10.2$ \\
\hline APACHE-II score, mean \pm SD & $18.8 \pm 7.3$ \\
\hline Other infections, $\mathrm{N}(\%)$ & $23(36.51 \%)$ \\
\hline Coinfection & $14(22.22 \%)$ \\
\hline \multicolumn{2}{|l|}{ Superinfection } \\
\hline $\begin{array}{l}\text { Vancomycin MIC levels, mean } \pm \text { SD (number of patients: } 60) \\
0.1, \mathrm{~N}(\%) \\
0.25, \mathrm{~N}(\%) \\
0.5, \mathrm{~N}(\%) \\
1, \mathrm{~N}(\%) \\
1.5, \mathrm{~N}(\%) \\
2, \mathrm{~N}(\%)\end{array}$ & $\begin{array}{l}0.93 \pm 0.48 \\
3(5.0 \%) \\
1(1.67 \%) \\
17(28.33 \%) \\
28(46.67 \%) \\
6(10.0 \%) \\
5(8.33 \%)\end{array}$ \\
\hline
\end{tabular}

SD: Standard deviation; COPD: Chronic obstructive pulmonary disease; AIDS: Acquired immune deficiency syndrome; ICU: Intensive care unit.

tors and the timing of positive cultures. Healthcare-associated infections could have community onset or hospital onset. The risk factors used by CDC are: 1) the presence of an invasive device at time of admission; 2) history of MRSA infection or colonization; 3) history of surgery, hospitalization, dialysis, or residence in a longterm care facility in the 12 months preceding cultures date. The presence of one or more of these risk factors defines community-onset healthcare-associated infections if the positive cultures were obtained $\leq 48$ hours of admission; while hospital-onset healthcare-associated infections have positive cultures obtained $>48$ hours after admission knowing that they might have $\geq 1$ risk factors. The CDC definitions of healthcare-associated infection are different than the definitions used in the current study since it combines hospital-acquired infections with healthcare-associated infections.

In earlier studies, the most common pathogens identified in HCAP were MRSA, Pseudomonas aeruginosa and other gram negative organisms [10,11]. However, no studies have specifically characterized HCAP caused by MRSA, even though it is a significant subgroup in this category due to the potential severe infection, drug resistance and high mortality.

In the present study, we attempted to understand this subgroup using a stringent definition of HCAP. In our study MRSA-HCAP accounted for $55.7 \%$ of all pneumonias, in comparison to $30.6 \%$ in a study by Micek, $\mathrm{T}$. et al. [12]. The increased rate in our study was surprising despite the similar settings of a tertiary-care teaching 
Table 2. Baseline characteristics comparison.

\begin{tabular}{|c|c|c|c|}
\hline Variable & Dead at 28 Days $(\mathrm{N}=18)$ & Alive at 28 Days $(\mathrm{N}=45)$ & p-Value \\
\hline Age (years) & $70.22 \pm 13.88$ & $64.24 \pm 15.74$ & $0.165(\mathrm{~T})$ \\
\hline APACHE II Score & $21.8 \pm 8.5$ & $17.6 \pm 6.5$ & $0.035(\mathrm{~T})^{*}$ \\
\hline Female Gender & $13(76.5 \%)$ & $24(53.3 \%)$ & $0.098(\mathrm{C})$ \\
\hline Antibiotic exposure 30 days prior to pneumonia diagnosis & $6(33.3 \%)$ & $14(31.1 \%)$ & $0.864(\mathrm{C})$ \\
\hline Hospitalization for greater than 5 days before treatment initiation & $1(5.6 \%)$ & $9(20.0 \%)$ & $0.257(\mathrm{~F})$ \\
\hline Bronchiectasis & $0(0.0 \%)$ & $4(8.9 \%)$ & $0.317(\mathrm{~F})$ \\
\hline Active Malignancy & $4(22.2 \%)$ & $6(13.3 \%)$ & $0.452(\mathrm{~F})$ \\
\hline AIDS & $1(5.6 \%)$ & $1(2.2 \%)$ & $0.493(\mathrm{~F})$ \\
\hline End stage Renal Disease & $4(22.2 \%)$ & $4(8.9 \%)$ & $0.210(\mathrm{~F})$ \\
\hline End stage Liver Disease & $4(22.2 \%)$ & $3(6.7 \%)$ & $0.095(\mathrm{~F})$ \\
\hline COPD & $8(44.4 \%)$ & $9(20.0 \%)$ & $0.063(\mathrm{~F})$ \\
\hline Use of Steroid & $0(0.0 \%)$ & $3(6.7 \%)$ & $0.551(\mathrm{~F})$ \\
\hline Heart Failure & $8(44.4 \%)$ & $13(28.9 \%)$ & $0.237(\mathrm{C})$ \\
\hline Vascular disease & $7(38.9 \%)$ & $19(42.2 \%)$ & $0.808(\mathrm{C})$ \\
\hline Diabetes Mellitus & $10(55.6 \%)$ & $15(33.3 \%)$ & $0.103(\mathrm{C})$ \\
\hline Multilobar Pneumonia & $8(44.4 \%)$ & $16(35.6 \%)$ & $0.512(\mathrm{C})$ \\
\hline De-escalation of antibiotic therapy & $11(55.0 \%)$ & $23(53.5 \%)$ & $0.911(\mathrm{C})$ \\
\hline Other Infections & $8(44.4 \%)$ & $15(33.3 \%)$ & $0.408(\mathrm{C})$ \\
\hline $\begin{array}{l}\text { Type of Other Infection } \\
\text { Coinfection } \\
\text { Superinfection }\end{array}$ & $\begin{array}{c}1(5.6 \%) \\
7(38.9 \%)\end{array}$ & $\begin{array}{l}8(17.8 \%) \\
7(15.6 \%)\end{array}$ & $0.120(\mathrm{~F})$ \\
\hline Blood culture positivity & $3(16.7 \%)$ & $6(13.3 \%)$ & $0.707(\mathrm{~F})$ \\
\hline $\begin{array}{l}\text { Specimen Source } \\
\text { Sputum } \\
\text { Tracheal Aspirate } \\
\text { BAL }\end{array}$ & $\begin{array}{c}15(83.3 \%) \\
3(16.7 \%) \\
0(0.0 \%)\end{array}$ & $\begin{array}{l}29(64.4 \%) \\
8(17.8 \%) \\
8(17.8 \%)\end{array}$ & $0.170(\mathrm{~F})$ \\
\hline $\begin{array}{l}\text { Treatment Type } \\
\text { Only Vancomycin } \\
\text { Only Linezolid } \\
\text { Switched between Vancomycin and Linezolid }\end{array}$ & $\begin{array}{c}12(66.7 \%) \\
1(5.6 \%) \\
5(27.8 \%)\end{array}$ & $\begin{array}{c}30(66.7 \%) \\
3(6.7 \%) \\
12(26.7 \%)\end{array}$ & $1.000(\mathrm{~F})$ \\
\hline $\begin{array}{l}\text { Vancomycin MIC } \\
0.1 \\
0.25 \\
0.5 \\
1 \\
1.5 \\
2 \\
\end{array}$ & $\begin{array}{c}1(5.9 \%) \\
1(5.9 \%) \\
4(23.5 \%) \\
7(41.2 \%) \\
1(5.9 \%) \\
3(17.6 \%)\end{array}$ & $\begin{array}{c}2(4.7 \%) \\
0(0.0 \%) \\
13(30.2 \%) \\
21(48.8 \%) \\
5(11.6 \%) \\
2(4.7 \%)\end{array}$ & $0.570(\mathrm{C}-\mathrm{A})$ \\
\hline
\end{tabular}

AIDS: Acquired immunodeficiency syndrome; COPD: Chronic obstructive pulmonary disease; BAL: Bronchoalveolar lavage; MIC: Minimum inhibiting concentration. $(\mathrm{T})=$ Two-Sample T-Test Result; $(\mathrm{C})=$ Chi-Square Test Result; $(\mathrm{F})=$ Fisher Exact Test Result; (C-A) $=$ Cochran-Armitage Trend Test Result. "Statistically Significant, $\mathrm{p}<0.05$.

Table 3. Multiple logistic regression result for predicting death at 28 days in HCAP patients.

\begin{tabular}{lcccc}
\hline \multicolumn{1}{c}{ Variable } & p-Value & Odds Ratio & Odds Ratio 95\% Confidence Limits \\
\hline Age & 0.196 & 1.043 & 0.978 & 1.113 \\
APACHE II Score & $0.026^{*}$ & 1.153 & 1.017 & 1.307 \\
Female Gender & 0.060 & 5.344 & 0.932 & 30.648 \\
End-Stage Liver Disease & 0.256 & 3.505 & 0.403 & 30.445 \\
COPD & $0.020^{*}$ & 7.869 & 1.387 & 44.646 \\
Diabetes Mellitus & 0.129 & 3.329 & 0.705 & 15.713 \\
Co-Infection (vs No Other Infections) & 0.432 & 0.364 & 0.029 & 4.534 \\
Super-Infection (vs No Other Infections) & $0.019^{*}$ & 14.057 & 1.532 & 128.982 \\
\hline
\end{tabular}

COPD: Chronic obstructive pulmonary disease; ${ }^{*}$ Statistically Significant, $\mathrm{p}<0.05$. 
hospital, similar sample size and selection criteria. This difference could be possibly explained by the different demographics between both studies. In general, a shift from hospital-acquired infection to HCAP is seen as a result of infection-control measures. Such measures are targeted to impact hospital-acquired infection.

In the current study, MRSA-HCAP seemed to have a predilection toward the elderly. We found that $88.9 \%$ had at least one comorbid condition, of which diabetes mellitus, heart failure, and vascular disease were the most common. All these conditions are commonly encountered in a tertiary-care center. All baseline characteristics were similar between survivors and nonsurvivors except APACHE II score (17. 6 vs 21.8, p value $<0.035$ ). Interestingly, APACHE II score, COPD and superinfection predicted 28-day mortality independently. These findings are consistent with previous studies [13-16]. The mean hospital length of stay was 19.1 days which is longer than what is observed with HCAP in general (18.7 days) [6]. The association with super infection was not seen before, but it is expected since other nosocomial infections might increase mortality. Other factors including age, previous antibiotics exposure and vancomycin MIC were not associated with mortality in contrast to previous studies [13-16].

HCAP usually requires mechanical ventilation in $44.5 \%$ of the cases [12]. In the current study, 41 patients (65.1\%) with MRSA-HCAP required mechanical ventilation compared to $60 \%$ in a different study by Shorr $\mathrm{AF}$ et al. which addressed 87 patients with HCAP due to MRSA [17]. The average ventilation duration $( \pm \mathrm{SD})$ was $7 \pm 10.2$ days in our study.

Vancomycin was used alone in the majority of our patients $(66.7 \%)$, only $6.3 \%$ were treated with linezolid alone and the remaining $27 \%$ were switched to or from linezolid based on the physician preference. The mean treatment duration $( \pm \mathrm{SD})$ was $14.6 \pm 7.8$ days which falls within the IDSA-recommended duration of 7 - 21 days. Vancomycin MIC was measured however it did not affect mortality which is not consistent with the literature [18]. This finding might be partially due to the use of linezolid in some patients in our study. Twenty-eight-day mortality rate of MRSA-HCAP in our study was $28.6 \%$ which is on the higher side of HCAP noted mortality $(11.4 \%$ - 34.1\%) [19,20].

There were several limitations to our study. It was a retrospective study, hence documentation error is possible. Being a single center study made it difficult to generalize the results to all healthcare settings. We were able to show the association between some risk factors and mortality; however the small sample size might have masked the influence of the other risk factors.

MRSA-associated HCAP is an important entity seen more commonly in elderly. It is associated with high morbidity and mortality. APACHE-II score, COPD and superinfection are independent risk factors for 28-day mortality. Increased awareness of HCAP epidemiology is needed to help design preventative measures. A prospective, multicenter, comparative study with a larger sample size is needed to establish a better characterization of this specific group of patients.

\section{REFERENCES}

[1] Rubinstein, E., Kollef, M.H. and Nathwani, D. (2008) Pneumonia caused by methicillin-resistant Staphylococcus aureus. Clinical Infectious Diseases, 46, S378-S385. doi:10.1086/533594

[2] American Thoracic Society (2005) Guidelines for the management of adults with hospital-acquired, ventilatorassociated, and healthcare-associated pneumonia. American Journal of Respiratory and Critical Care Medicine, 171, 388-416.

[3] Ewig, S., Welte, T., Chastre, J. and Torres, A. (2010) Rethinking the concepts of community-acquired and healthcare-associated pneumonia. The Lancet Infectious Diseases, 10, 279-287. doi:10.1016/S1473-3099(10)70032-3

[4] Brito, V. and Niederman, M.S. (2009) Healthcare-associated pneumonia is a heterogeneous disease, and all patients do not need the same broad-spectrum antibiotic therapy as complex nosocomial pneumonia. Current Opinion in Infectious Diseases, 22, 316-325. doi:10.1097/QCO.0b013e328329fa4e

[5] Zilberberg, M.D. and Shorr, A.F. (2009) Epidemiology of healthcare-associated pneumonia (HCAP). Seminars in Respiratory and Critical Care Medicine, 30, 10-15. doi:10.1055/s-0028-1119804

[6] Venditti, M., Falcone, M., Corrao, S., Licata, G., Serra, P. and Study Group of the Italian Society of Internal Medicine (2009) Outcomes of patients hospitalized with community-acquired, health care-associated, and hospital-acquired pneumonia. Annals of Internal Medicine, 150, 1926.

[7] Eber, M.R., Laxminarayan, R., Perencevich, E.N. and Malani, A. (2010) Clinical and economic outcomes attributable to health care-associated sepsis and pneumonia. Annals of Internal Medicine, 170, 347-353. doi:10.1001/archinternmed.2009.509

[8] Chalmers, J.D., Taylor, J.K., Singanayagam, A., Felming, G.B., Akram, A.R. and Mandal, P., et al. (2011) Epidemiology, antibiotic therapy, and clinical outcomes in health care-associated pneumonia, a UK cohort study. Clinical Infectious Diseases, 53, 107-113. doi:10.1093/cid/cir274

[9] Polverino, E. and Torres, A. (2009) Current perspective of the HCAP problem, is it CAP or is it HAP? Seminars in Respiratory and Critical Care Medicine, 30, 239-248. doi:10.1055/s-0029-1202940

[10] Kollef, M.H., Shorr, A., Tabak, Y.P., Gupta, V., Liu, L.Z. and Johannes, R.S. (2005) Epidemiology and outcomes of health-care-associated pneumonia, results from a large US database of culture-positive pneumonia. Chest, 128, 
3854-3862. doi:10.1378/chest.128.6.3854

[11] Chroneou, A., Zias, N., Beamis Jr., J.F. and Craven, D.E. (2007) Healthcare-associated pneumonia, principles and emerging concepts on management. Expert Opinion on Pharmacotherapy, 8, 3117-3131.

[12] Micek, S.T., Kollef, K.E., Reichley, R.M., Roubinian, N. and Koleff, M.H. (2007) Health care-associated pneumonia and community-acquired pneumonia: A single-center experience. Antimicrobial Agents and Chemotherapy, 51, 3568-3573.

[13] Carratala, J., Mykietiuk, A., Fernandez-Sabe, N., Suarez, C., Dorca, J. and Verdaquer, R., et al. (2007) Health careassociated pneumonia requiring hospital admission, epidemiology, antibiotic therapy, and clinical outcomes. Archives of Internal Medicine, 167, 1393-1399. doi:10.1001/archinte.167.13.1393

[14] Shindo, Y., Sato, S., Maruyama, E., Ohashi, T., Oqawa, M. and Hashimoto, N., et al. (2009) Health-care-associated pneumonia among hospitalized patients in a Japanese community hospital. Chest, 135, 633-640. doi:10.1378/chest.08-1357

[15] Kanerva, M., Ollgren, J., Virtanen, M.J., Lyytikainen, O. and Prevalence Survey Study Group (2008) Risk factors for death in a cohort of patients with and without healthcare-associated infections in Finnish acute care hospitals. Journal of Hospital Infection, 70, 353-360. doi:10.1016/j.jhin.2008.08.009

[16] Sund-Levander, M., Ortqvist, A., Grodzinsky, E., Klefsqard, O. and Wahren, L.K. (2003) Morbidity, mortality and clinical presentation of nursing home-acquired pneumonia in a Swedish population. Scandinavian Journal of Infectious Diseases, 35, 306-310. doi:10.1080/00365540310008456

[17] Shorr, A.F., Haque, N., Taneja, C., Zervos, M., Lamerato, L., Kothari, S., et al. (2010) Clinical and economic outcomes for patients with health care-associated Staphylococcus aureus pneumonia. Journal of Clinical Microbiology, 48, 3258-3562. doi:10.1128/JCM.02529-09

[18] Haque, N.Z., Zuniga, L.C., Peyrani, P., Reyes, K., Lamerato, L., moore, C.L., et al. (2010) Relationship of vancomycin minimum inhibitory concentration to mortality in patients with methicillin-resistant Staphylococcus aureus hospital-acquired, ventilator-associated, or healthcare-associated pneumonia. Chest, 138, 1356-1362. doi:10.1378/chest.09-2453

[19] Jeon, E.J., Cho, S.G., Shin, J.W., Kim, J.Y., Park, I.W. and Choi, B.W., et al. (2011) The difference in clinical presentations between healthcare-associated and communityacquired pneumonia in university-affiliated hospital in Korea. Yonsei Medical Journal, 52, 282-287. doi:10.3349/ymj.2011.52.2.282

[20] Tasbakan, M.S., Bacakoglu, F., Basogu, O.K., Gurgun, A., Basarik, B., Citim Tuncel, S., et al. (2011) The comparison of patients with hospitalized health-care associated pneumonia to community-acquired pneumonia. Tüberküloz ve Toraks Dergisi, 59, 348-354. doi:10.5578/tt.2359 